\title{
INTRODUCGIÓN AL TRATAMIENTO BASADO EN LA MENTALIZACIÓN PARA EL TRASTORNO LÍMITE DE LA PERSONALIDAD \\ INTRODUCTION TO THE MENTALIZATION-BASED TREATMENT FOR BORDERLINE PERSONALITY DISORDER
}

\author{
Sabrina Sánchez Quintero a E Irene de la Vega ${ }^{\mathrm{b}}$ \\ a Psicóloga Clínica. Centro de Atención Integral a Drogodependientes CAID Sur, de la Comunidad de \\ Madrid.sabrinasanchez1984@hotmail.com \\ b Psicóloga Clínica. Hospital de Día de Trastornos de Personalidad. Hospital Clínico San Carlos, Madrid.
}

Cómo referenciar este artículo/How to reference this article:

Sánchez-Quintero, S. y De la Vega, I. (2013). Introducción al tratamiento basado en la mentalización para el trastorno límite de la personalidad [Introduction to the mentalization-based treatment for borderline personality disorder]. Acción Psicológica, 10(1), 21-32. http://dx.doi.org/10.5944/ap.10.1.7030

\section{Resumen}

La terapia basada en la mentalización (TBM) para el Trastorno Límite de Personalidad (TLP) ha sido desarrollada principalmente por Anthony Bateman y Peter Fonagy. La mentalización es el proceso mediante el cual entendemos a los otros y a nosotros mismos en términos de estados subjetivos (deseos, pensamientos, sentimientos), y la estrecha relación de nuestras conductas con los mismos. Esta capacidad no es innata: debe desarrollarse en un contexto de apego seguro. Según este modelo, los síntomas del TLP resultan del re-surgimiento de modos pre-mentalizadores de funcionamiento psíquico tras una supresión parcial y específica de la mentalización, ocurrida en un contexto de hiperactivación del sistema de apego. En la Unidad de Hallwick (Hospital St. Ann, Londres), dirigida por Bateman, el tratamiento se estructura en tres fases, centrándose en favorecer la mentalización. Su efectividad en la reducción de los síntomas borderline ha quedado demostrada en ensayos controlados aleatorizados que justifican su utilidad.

Palabras clave: trastorno límite de la personalidad; mentalización; apego; Hallwick; función reflexiva.

\section{Abstract}

Mentalization based treatment (MBT) for Borderline Personality Disorder (BPD) has been developed mainly by Anthony Bateman and Peter Fonagy. Mentalization is the process by which we understand others and ourselves in terms of subjective states (wishes, thoughts, feelings) and the close link between these and our behaviors. Such ability is not innate: it must develop in a secure attachment context. According to this model, the BPD symptoms result from the reemergence of pre-mentalizing modes of psychic functioning, after a partial and specific suppression of mentalizing in a context of hyperactiva- 
tion of the attachment system. In Hallwick Unit (Hospital St. Ann, London), conducted by Bateman, treatment is structured in three phases, keeping focus on enhancing mentalization. It has been proved effective in reducing borderline symptoms in randomized controlled trials, which support its usage.

Keywords: borderline personality disorder; mentalization, attachment; Hallwick; reflective function.

\section{Introducción}

La terapia basada en la mentalización (TBM) es una terapia de corte psicodinámico, sustentada sobre todo en la teoría del apego (Bowlby, 2011) y desarrollada especialmente para el abordaje integral del Trastorno Límite de la Personalidad (TLP). Sus autores principales son Anthony Bateman y Peter Fonagy (Cuevas y López, 2012), si bien también han surgido otros nombres importantes como el de Jon Allen (2006). Desafortunadamente, sus manuales no han sido traducidos al castellano, lo que puede haber contribuido a su limitada difusión en nuestro país.

El conocimiento que tenemos sobre la relevancia de la teoría del apego tanto en el desarrollo del TLP como en el tratamiento del mismo no es algo nuevo (Keinänen, Johnson, Richards y Courtney 2012; Nickell,Waudby y Trull, 2002). En estudios realizados con la Entrevista de Apego del Adulto (AAI) (George, Kaplan y Main, 1985) ha quedado demostrado que las narrativas de los pacientes con TLP reflejan una mayor prevalencia de apego inseguro, más concretamente, del estilo desorganizado (Fonagy et al., 1996; Fonagy, Target y Gegerly, 2000; Gunderson y Lyons-Ruth, 2008). Sin embargo, de acuerdo con Bateman y Fonagy (2004), el simple establecimiento de esta relación entre TLP y apego desorganizado no explica la génesis del trastorno límite de personalidad.

Estos autores ponen el acento en la capacidad de mentalización como variable fundamental a la hora de entender y abordar la patología borderline (Bateman y Fonagy, 2004, 2006, 2009, 2010). El término de «mentalización», que en la mayoría de las ocasiones, equivale o sustituye al de "función reflexiva» (Bouchard et al., 2008; Fonagy, Target, Steele y Stelle, 1997; Lanza Castelli, 2011), hace referencia al proceso psicológico mediante el cual percibimos e interpretamos la conducta humana, tanto la propia como la ajena, como un elemento dependiente o relacionado con estados mentales subjetivos e intencionales, tales como los deseos, los pensamientos o sentimientos (Allen y Fonagy, 2006). Bateman y Fonagy (2004, 2006, 2010) consideran que la disfunción en la capacidad de mentalización es un elemento clave en el TLP y, por tanto, su trabajo terapéutico se centra en intentar optimizar y favorecer el uso de dicha capacidad, en un contexto de apego seguro. En el presente trabajo intentaremos resumir los principales puntos teórico-prácticos del enfoque del tratamiento basado en la mentalización para el TLP.

\section{Concepto de «mentalización»}

«Mentalizar» puede definirse como la capacidad para "percibir e interpretar la conducta como estrechamente relacionada con estados mentales intencionales», y se basa en el supuesto de que nuestros estados mentales influyen en nuestra conducta (Bateman y Fonagy, 2006).

A grandes rasgos, la mentalización es una capacidad que nos permite tener una representación de nosotros mismos, de nuestro self, como un «agente», es decir, nos permite sentirnos dueños de nuestras conductas y pensamientos. Al contrario de lo que ha afirmado durante siglos la tradición cartesiana, la capacidad implícita en la frase "pienso, luego existo», no es innata, no nos viene dada por el hecho de ser humanos. Es un logro, el resultado de un proceso evolutivo que, desgraciadamente, puede torcerse en determinadas circunstancias (Bateman y Fonagy, 2004). Además, la capacidad de mentalización nos permite interpretar las conductas de los otros en función de sus estados mentales (creencias, sentimientos, deseos, etc.). La capacidad de interpretar a los otros en términos psicológicos, llamada por estos autores «función interpersonal interpretativa» (FII) (op. cit p.58) requiere de una robusta 
actividad mentalizadora y es un proceso eminentemente social, que precisa, para su desarrollo, de la proximidad de una figura de apego durante los primeros años de vida. Asimismo, una adecuada capacidad para mentalizar es fundamental para la regulación de nuestras emociones (Bateman y Fonagy, 2006). Alguien con una débil capacidad de mentalización resulta mucho más vulnerable a los cambios y a las presiones que se produzcan en el entorno, como puede sucederle a un individuo con TLP.

Las disfunciones en la mentalización pueden dar lugar a malinterpretaciones de los estados mentales, las cuales a su vez pueden derivar en los problemas propios del TLP, tanto a nivel de relaciones interpersonales, como a nivel de malestar psíquico y a alteraciones conductuales características, tales como los intentos de suicidio o las auto/heteroagresiones. Sin embargo, Bateman y Fonagy (2006) no consideran que los déficit en la capacidad de mentalización sean exclusivos de los individuos con TLP ya que, potencialmente, todos los trastornos mentales pueden concebirse como un trastorno en la mentalización, esto es, como un trastorno consistente en «una mente que malinterpreta su propia experiencia de sí misma» (p. 8). No obstante, y esto es un punto central de su teoría, consideran que no en todos los casos es tan productivo o necesario centrarse, de cara al tratamiento, en el aumento de la capacidad mentalizadora como en el caso de los individuos con personalidad límite.

\section{¿Cómo se desarrolla normalmente la capacidad de mentalización en un contexto de apego seguro?}

Como hemos explicado anteriormente, un bebé carece de una representación subjetiva y coherente de sí mismo, y no es capaz de distinguir sus estados mentales de la realidad, ni de percibirse como un self «agente» (no ha desarrollado una representación del "sí mismo» como dueño consciente de sus actos, o como «pensador» de sus propios pensamientos). El desarrollo de dicha representación requiere, al principio de la vida, que los otros le perciban como una persona que piensa, siente y hace (como un self agente) y él, a su vez, sea consciente de que los otros le perciben como tal (Fonagy, 1991).

El desarrollo de este self agente se produce en un contexto que permita al niño regular sus afectos. En el caso de que se den las condiciones necesarias, el niño atraviesa una serie de estadios: éstos van desde el momento en que el niño se da cuenta de que, mediante sus acciones, puede influir físicamente sobre el entorno (esto es, se percibe como un agente "físico»), lo cual ocurre antes de los tres meses de edad, hasta el quinto estadio (que se inicia alrededor de los 3-4 años) en que el niño logra percibirse como un "agente representacional», cuando entiende que sus acciones están guiadas por sus estados mentales (deseos, creencias...) y que estos son suyos, independientes y opacos para el resto de las personas, ya que son representacionales por naturaleza. Es capaz de identificar y etiquetar sus sentimientos y sus pensamientos. Además, puede relacionar sus recuerdos de acciones intencionales para adquirir un sentido de la identidad coherente (Bateman y Fonagy, 2004).

Por razones de espacio no podemos extendernos en la explicación de todas las fases evolutivas en el desarrollo de la capacidad «agentiva» del self. Remitimos al lector interesado en el conocimiento de estas fases evolutivas al tercer capítulo del manual de Bateman y Fonagy (2004). Únicamente haremos referencia a dos modos de comprender los estados mentales que anteceden a la mentalización, ya que son de crucial importancia para la comprensión de algunos aspectos del funcionamiento mental de los pacientes con TLP. Estos dos modos primitivos pre-mentalizadores de funcionamiento psíquico son la «equivalencia psíquica» y el "modo aparentado»" (Fonagy y Target, 1996; Bateman y Fonagy 2004, 2006).

* No hemos encontrado una traducción plenamente satisfactoria para el concepto "pretend mode». Pretend puede entenderse como «aparentar», «fingir», «de mentira» (como en los juegos infantiles) o «hacer un como si». En un trabajo en castellano sobre TBM hemos encontrado «hacer de cuenta» (Lanza Castelli, 2011) y nos consta que algunos profesionales hablan del modo «como si» (Mas, 2012). Sin embargo, nos parece que el término «modo aparentado» recoge mejor la esencia del concepto de «pretend mode» y por eso lo utilizamos aquí. 
En el modo de «equivalencia psíquica», la realidad interna y la externa son isomórficas. Lo que existe en la mente, existe en el mundo externo y viceversa. La fantasía se siente como tremendamente real, lo cual puede generar un intenso malestar. Bateman y Fonagy (2004, p. 69) nos ponen como ejemplo la historia de un niño de tres años que, disfrazado de Batman, huyó aterrorizado al mirarse al espejo. Aquí, el considerar la fantasía y la realidad como equivalentes no supuso más que una divertida anécdota (no tan graciosa para el niño, quizás, pero sí para su entorno). Sin embargo, en el caso de un individuo adulto funcionando en el modo de equivalencia psíquica, puede dar lugar a catastróficas consecuencias, como veremos en el siguiente apartado.

Por otro lado, el «modo aparentado» supone lo contrario: hay una disociación total entre los estados mentales y la realidad física, entre lo interno y lo externo. Los mismos autores ponen otro ejemplo, en el cual un niño de dos años y medio jugaba empujando una silla tumbada y disparando a un enemigo imaginario. No obstante, al preguntarle al niño si se trataba de una silla o un tanque, inmediatamente puso la silla en su posición, abandonando el juego. En este modo de funcionamiento, no puede haber ninguna conexión entre lo que se finge y la realidad.

La mentalización se logra al integrar ambos modos de representación, de tal manera que los estados mentales representan con bastante precisión la realidad, pero al mismo tiempo se mantienen separadas de la misma (Bateman y Fonagy, 2006); es decir, se sitúan en un nivel de abstracción superior a la realidad concreta, pero no en un plano de fantasía totalmente alejado de la realidad.

En el siguiente apartado volveremos sobre estos dos modos de experiencia subjetiva, dada su gran implicación en el funcionamiento borderline.

Volvamos al desarrollo normal de la mentalización y de las representaciones del self ¿Cuáles son las condiciones contextuales «necesarias» para este desarrollo de una representación del self subjetivamente coherente, la cual constituye, a su vez, una condi- ción sine qua non para una adecuada mentalización?

Hemos de tener en cuenta que en un primer momento, los humanos no somos conscientes de nuestros estados emocionales. Ni siquiera somos capaces de diferenciarlos: somos un manojo de señales fisiológicas indiferenciadas. Un bebé siente miedo, pero no es capaz de identificarlo ni de etiquetarlo como tal porque carece de un sistema conceptual en el que tal emoción esté representada. De bebés, aprendemos a diferenciar nuestros estados afectivos a través de las señales retroalimentadoras que nos envían nuestras figuras de apego ante nuestra expresión de emociones (Gegerly y Watson, 1996). En otras palabras, cuando el niño pequeño emite una señal que exprese algún afecto (por ejemplo, enfado), el padre o madre responden especularizando, o reflejando, dicho afecto, de manera contingente y congruente con dicho afecto. Sin embargo, dicho reflejo debe ser marcado, es decir, exagerado o dramatizado, para que el niño pueda entender que no se trata del afecto de la madre el que está viendo reflejado en su rostro, sino una imitación del suyo propio (Bateman y Fonagy, 2004, 2006).

Este reflejo contingente que el cuidador realiza, a modo de espejo, de las emociones del niño ya ha sido descrito como una fase clave en el desarrollo del self (Winnicott, 1982). Ejerce un papel fundamental en la regulación de sus emociones, ya que cuando la madre remeda la expresión de las emociones de su bebé, de algún modo las atempera. Sin embargo, si este reflejo no es marcado, el niño no atribuirá esa emoción a sí mismo, sino a su madre, como si fuera real. De este modo, lo que se produce es un aumento de esa emoción, especialmente cuando se trata de malestar. El reflejo no marcado, por tanto, no ejercería esa función regulatoria. Algunos estudios revelan que la ausencia de esta especularización contingente, congruente y marcada de la expresión del niño se asocia con un desarrollo posterior de un estilo de apego desorganizado (Bateman y Fonagy, 2004, 2006).

Esta especularización es fundamental, por tanto, por dos motivos. Por un lado, la res- 
puesta de una figura de apego ante su malestar le permite adquirir cierto sentido de "control» y de autoeficacia, al ser capaces de obtener una respuesta contingente a su expresión afectiva. Y por otro lado, la internalización de la respuesta reflejo de la madre dará lugar a la representación simbólica de ese estado interno. El cuidador, de este modo, moldea y da significado a los estados afectivos del niño, proporcionándole las representaciones que formarán el núcleo de su sentido del self (la representación mental que el cuidador tiene de la mente del niño será interiorizada e integrada y así, el niño logrará una realidad psíquica en la que su representación del self esté claramente separada de su representación del otro). (Bateman y Fonagy, 2004).

En su principal trabajo sobre la TBM, Bateman y Fonagy (2004), aportan una gran cantidad de evidencia sobre la relación entre la función reflexiva de las figuras de apego y la capacidad de mentalización que desarrollan los hijos. En varios estudios controlados (para una revisión, ver la obra citada), se ha visto que las madres que utilizan vocabulario referente a estados mentales y generan un contexto de apego seguro (con interacciones lúdicas y desenfadadas con sus bebés) dan lugar a una buena función interpersonal interpretativa (FII) y una adecuada capacidad para regular sus emociones, que permite a los niños protegerse mejor de desafíos ambientales posteriores. Por el contrario, un sistema de apego deficitario constituirá un importante factor de vulnerabilidad que, en presencia de ciertos estresores, podría dar lugar a una grave patología de la personalidad.

\section{EI TLP desde el punto de vista de la Terapia Basada en la Mentalización}

Como factores de vulnerabilidad para un ulterior desarrollo de un TLP, entenderíamos cualquier circunstancia que impidiera la emergencia de una representación del self coherente e integrada, para la cual es necesaria una robusta capacidad para mentalizar. Recordemos que, para que esto se produzca, es precisa una especularización o reflejo congruente y marca- do de las expresiones del niño, para que éste pueda elaborar en su mente unas representaciones de dichos afectos. Si dicho reflejo es congruente, pero no marcado, el niño atribuirá el afecto a su cuidador, con lo que dichas representaciones no se establecerán porque el niño no puede dar un significado a su experiencia y porque se producirá además a una escalada en el afecto negativo, no logrando el objetivo de regular y atemperar tal manifestación afectiva. Este patrón relacional, mantenido en el tiempo, podría dar lugar a la identificación proyectiva, la cual constituye el principal mecanismo de defensa del TLP (Kernberg, 1999) y favorece la persistencia de la equivalencia psíquica como modo de experimentar la realidad interna, ya que, en este caso, ésta se correspondería con lo observado en el exterior, y por tanto, se viviría como si fuera $100 \%$ real.

Por otro lado, si la especularización no es congruente con el afecto del niño, es decir, no refleja de manera más o menos precisa la emoción que el niño está expresando, éste no podrá establecer ningún vínculo entre sus estados mentales y la realidad que observa. No siente que la expresión del adulto tenga algún tipo de relación con él. Por tanto, las representaciones de los afectos que se generen en este niño no tendrán ningún peso a nivel emocional. Las etiquetas referidas a emociones que aprenda posteriormente no se corresponderán con estados mentales «reales». En este caso hablaríamos de un «modo aparentado» de funcionamiento psíquico, que subyace al discurso «pseudo-mentalizador» que encontramos en muchos pacientes con TLP. La pseudo-mentalización, en terapia, puede dar lugar a eternas charlas improductivas sobre emociones, de tal manera que el paciente podría parecernos capaz de mentalizar. No obstante, todas esas etiquetas «enlatadas» utilizadas por el paciente no tienen ninguna conexión con sus sentimientos reales, para los cuales no tiene un sistema simbólico representacional adecuado que le permita entenderlos y manejarlos (Bateman y Fonagy, 2006). De ahí que también se emplee el vocablo "como si» para referirse a este tipo de funcionamiento, ya que el paciente habla de sus emociones "como si» las estuviese experimentando realmente. 
Idealmente, estos aprendizajes se dan en un contexto de apego seguro y en un ambiente de interacción lúdica entre el niño y sus cuidadores. La ausencia de un sentido del self estable, agente, está íntimamente ligada con el patrón de apego desorganizado (Lyons-Ruth, 1996), caracterizado sobre todo por miedo al cuidador y una falta de coherencia en la estrategia de apego. Cuando el niño carece del reflejo adecuado de su propia experiencia, y por tanto, no puede desarrollar una representación de su self, se ve obligado internalizar representaciones incongruentes, provenientes de su figura de apego, como parte de su representación del self (Bateman y Fonagy, 2004). Los autores denominan a esta representación «self ajeno» ${ }^{* *}$ o alien self, una representación dentro del self que genera una incongruencia dentro de la representación global del self, dando como resultado una sensación de tener pensamientos o ideas que el individuo «sabe» que son suyas, pero no «siente» como suyas.

Es posible que todos tengamos, dentro de nuestro self, ciertos estados mentales que no logremos integrar coherentemente dentro de nuestra estructura. Eso es debido a que, incluso en contextos de apego seguro, con figuras parentales atentos a las señales emitidas por sus hijos, la capacidad mentalizadora parental que permite detectar la emoción y reflejarla congruente y marcadamente no siempre es perfecta. En estos casos, en que esta «especularización» sea bastante contingente con la expresión afectiva del bebé, es posible que todos interioricemos ciertas representaciones del otro que creen cierta "discontinuidad» dentro de nuestro self. No obstante, si desarrollamos una capacidad robusta de mentalización, estas incoherencias pueden integrarse perfectamente sin generar ningún tipo de malestar. Cuando existe una historia de apego desorganizado, este "alien self» interiorizado es bastante más amplio y si además el individuo no puede hacer uso de la mentalización para integrarlo dentro de una estructura coherente, será tremendamente difícil para él desarrollar una

\footnotetext{
** Alien self es el término que aparece en los textos originales de Bateman y Fonagy (2004, 2006). Lo conservamos en su forma original por haberse extendido entre profesionales españoles (Mas, 2012).
}

sentido de identidad propia. Los autores describen las conductas «manipuladoras» de los pacientes con TLP como un intento de restaurar la coherencia del self mediante «identificación proyectiva».(Bateman y Fonagy, 2004, 2006). Cuando el self detecta que existen sentimientos y pensamientos propios (parte de este «alien self»), que no vive como reales, manipula inconscientemente a la figura de apego hasta que ésta tenga las emociones que él mismo tiene contra sí mismo. Así, logra externalizarlas y se restaura, de algún modo, la deseada coherencia interna. Lo hace así porque no puede hacerlo de otro modo, porque carece de la posibilidad de mentalizar estas experiencias internas. Y además, suele resultar contraproducente, ya que la respuesta del otro, especialmente si es una figura de apego, puede ser también de intenso enfado y en estas circunstancias, decrece también su capacidad reflexiva, lo cual repercute, si se mantiene en el tiempo, en la función mentalizadora del niño. Aún más dramático resulta en los casos de niños que han resultado víctimas de maltrato o negligencia. Aquí, internalizan, como parte de la estructura de su self, además de representaciones incoherentes, una representación maltratadora. Posteriormente, la experiencia de «auto-maltrato» que esto puede sostener crea un estado mental abrumador e insoportable, de la que deben liberarse para no atacarse a sí mismos, mediante estas conductas "controladoras» O «manipuladoras» que solemos identificar en los pacientes con TLP. Como bien apuntan Bateman y Fonagy (2006) «la identificación proyectiva en tales casos se vive como esencial para la supervivencia» (p. 17).

Desde este enfoque, los síntomas del TLP se entienden como resultado de una supresión parcial y específica de la mentalización. Los pacientes borderline no muestran una capacidad nula para la comprensión de sus estados mentales en todo momento. Pero sí suelen fracasar en este sentido cuando se encuentran en una situación de elevada activación emocional, especialmente en el contexto de relaciones de apego intensas, y ocurre con mayor probabilidad cuando hay un trasfondo traumático en la historia biográfica del individuo. Quizá se trate, dicen Bateman y Fonagy (2004), de una estrategia 
defensiva aprendida en la primera infancia. El niño aprende que es preferible evitar pensar sobre estados mentales del otro, simplemente porque debe de ser insoportable pensar sobre los estados mentales de alguien que le maltrata, cuando debería estar cuidándole y protegiéndole. Además, se da una situación paradójica, y es que, dado que el sistema de apego (búsqueda de protección por parte del cuidador) se activa ante el peligro percibido, y la persona a la que recurrimos buscándolo es la misma que nos maltrata, dicho sistema de apego puede estar permanentemente activado, en una especie de círculo vicioso ( «cuanto más me maltratas, más miedo siento, y cuanto más miedo siento, más te busco»). Esto podría explicar por qué, posteriormente, muchos de estos pacientes se mantienen en relaciones tormentosas con parejas maltratadoras, a las que no abandonan pese al daño que saben que les hacen.

La hiperactivación del sistema de apego, que le lleva a buscar la proximidad de una figura de apego maltratante, y que resulta de estas experiencias tempranas, pueden llegar a inhibir las estructuras cerebrales implicadas en la mentalización (área frontal).

A su vez, la «supresión parcial» de la mentalización puede dar lugar al re-surgimiento de los modos pre-mentalizadores de subjetividad: la equivalencia psíquica y el modo aparentado, los cuales a su vez favorecen la aparición de manifestaciones conductuales típicas del TLP. En sus dos manuales de referencia (Bateman y Fonagy; 2004, 2006) los autores nos proporcionan explicaciones detallada de su forma de comprender la sintomatología borderline desde el «modelo de supresión de la mentalización». Por ejemplo, deducimos que el paciente se encuentra funcionando en el modo de «equivalencia psíquica», cuando muestra la ideación paranoide: como está absolutamente convencido de que lo que está en su mente equivale a la realidad y no acepta interpretaciones alternativa, si lo que hay en su mente es un modelo de relación maltratante, tendrá plena seguridad en que en el otro le está maltratando. La autolesión también puede explicarse así: las partes del cuerpo agredidas equivaldrían a estados mentales que nos resultan insoportables. Atacando a esas partes del cuerpo, estamos inten- tando erradicar ese malestar que no podemos mentalizar. Por otro lado, recordemos que el funcionamiento en "modo aparentado» da lugar a la pseudo-mentalización. En terapia, podemos verlo porque el paciente emite un discurso aparentemente mentalizador (usando múltiples términos que hacen referencia a sentimientos, pensamientos, deseos...), pero realmente "habla sin decir nada», y son sesiones aparentemente productivas pero el tiempo pasa sin progresar realmente; el terapeuta se queda con la sensación de que no puede llegar a conectar con lo que le dice el paciente. Instamos al lector interesado a que consulten la guía práctica de Bateman y Fonagy (2006) para una explicación sencilla de otros síntomas desde el punto de vista de este modelo, que nosotros por falta de espacio no podemos abordar aquí.

Cuando el terapeuta detecta señales de que el paciente no mentaliza, los autores aconsejan explorar los antecedentes del fallo, sin interpretar nada más allá de lo que el paciente nos cuenta. Si damos por hecho que «sabemos» lo que el otro piensa, caemos en una postura «nomentalizadora». Los estados mentales del otro son deducibles, pero no adivinables. Es por ello que en fases iniciales o en momentos de nomentalización, al contrario que otros autores (Clarkin, Yeomans y Kernberg, 2006), Bateman y Fonagy consideran que realizar interpretaciones puede resultar inútil o contraproducente. La TBM pretende ayudar al paciente a incrementar su capacidad de mentalización, mediante la exploración e identificación de emociones en multiplicidad de situaciones. Se trata de estimular una relación de apego lo suficientemente significativa como para que el paciente se implique en terapia, pero al mismo tiempo ayudándole a mantenerse en una postura mentalizadora (Bateman y Fonagy, 2006)

\section{Tratamiento del TLP desde la TBM: organización del tratamiento}

En la Unidad de Halliwick (que dirige el Dr. Bateman en el hospital de St. Ann, en Londres) se sigue el «modelo de equipo único» ${ }^{* * *}$, un

\footnotetext{
*** One-team model.
} 
equipo interdisciplinar en el que todas las decisiones se toman en conjunto El objetivo es evitar las «escisiones» por parte de los pacientes, entre los miembros del equipo terapéutico «buenos» y los «malos». Las responsabilidades son asumidas por todo el personal.

Se combina terapia individual y grupal en todos los casos, no primándose una por encima de la otra. Las dificultades, ansiedades y miedos que puedan surgir en los grupos pueden ser tratadas en las sesiones individuales como ejemplo de situaciones interpersonales a trabajar. Por otra parte, los autores son conscientes de que la atención sanitaria difiere en función del sistema de salud de cada país y consideran que las directrices generales del modelo pueden ser aplicadas y adaptadas a las exigencias del contexto. En Halliwick cuentan con dos programas: el hospital de día, por un lado, y el Programa Intensivo Ambulatorio (Intensive Out-patient Programme: IOP). En éste último, la terapia individual y en pequeños grupos constituyen los elementos fundamentales; si bien, también se procura que los pacientes acudan a sesiones de terapia ocupacional y expresiva, sin obligarles a ello. Consiste en una sesión individual de 50 minutos y una grupal de 90 a la semana. En el caso del Hospital de Día, los pacientes acuden a diario y son divididos, de cara a la terapia grupal, en pequeños grupos, si bien existen sesiones "grupo grande» de carácter bimensual. Lo más característico del hospital de día es el carácter «holístico» de la intervención, es decir, la relación entre todos los componentes. De esta manera, los asuntos que emergen en las diferentes sesiones terapéuticas deben ser abordados y conocidos por el equipo -para lo cual se establecen reuniones periódicas- $\mathrm{y}$, de ser necesario, tenerlas en cuenta para el resto de intervenciones. De ahí que los autores señalen la capacidad para el trabajo en un equipo multidisciplinar como una habilidad fundamental del terapeuta, entre otras (Bateman y Fonagy, 2004). Un equipo cohesionado, si ya es deseable para trabajar con cualquier patología mental grave, se convierte en absolutamente fundamental en el caso de los pacientes con TLP. Es necesaria la comunicación fluida entre los distintos profesionales (psiquiatras, psicólogos, terapeutas ocupacionales y enfermeros), siguiendo una estrategia coherente. Para cada paciente existiría un profesional de referencia, casi siempre el terapeuta individual.

El tratamiento se estructura en tres grandes fases, cada una con su propio objetivo y procesos específicos (Bateman y Fonagy, 2006). En la primera fase («fase inicial») se intenta lograr que el paciente se involucre en el tratamiento. En ella, se evalúa la capacidad de mentalización del paciente mediante el análisis conjunto de las relaciones interpersonales del paciente y explorando atentamente cómo relata los eventos situacionales pasados y presentes, los sentimientos y pensamientos que el paciente tuvo, los estados mentales atribuidos al otro, cómo entiende los propios actos y en el caso de sospechar que el paciente esté "pseudo-mentalizando», se puede recurrir a preguntas que los autores llaman «contrafactuales» (en las que se le pide que contemple una idea totalmente opuesta a la que estaba considerando) y observando la flexibilidad con la que responde a la misma. Asimismo, se analiza el patrón de relaciones interpersonales que el paciente suele establecer. Concluye con la formulación del caso. En la fase media tiene lugar el trabajo más duro para el paciente, pues incluye terapia individual y grupal encaminada a trabajar la mentalización (mediante técnicas específicas, de profundidad creciente) mientras el terapeuta debe luchar para controlar la contratransferencia y mantener la alianza terapéutica. Durante la fase final (generalmente tras 12-18 meses) se prepara al paciente para la finalización del tratamiento intensivo, trabajando los sentimientos de pérdida y el mantenimiento de los logros.

\section{Terapia basada en la mentalización: estrategias generales de tratamiento}

Como ya hemos dicho, el objetivo que subyace a toda intervención en pacientes con TLP desde este modelo es el de favorecer la capacidad de mentalización, necesaria para lograr un sentido del self, de la identidad, más fuerte y seguro (Bateman y Fonagy, 2004). Se trata de que el paciente «averigüe más sobre cómo 
piensa y siente sobre sí mismo y los demás, cómo esto gobierna sus respuestas a los otros y cómo los "errores» en la comprensión de sí mismo y de los otros le llevan a emprender acciones en un intento de mantener la estabilidad y dar sentido a sentimientos incomprensibles» (Bateman y Fonagy, 2006, p. 37). Para lograrlo, Bateman y Fonagy (2004) sugieren cuatro estrategias generales, que describiremos someramente a continuación:

a) Favorecer la mentalización: para ello, el terapeuta debe tratar de mantenerse en una posición «mentalizadora», reflexionando sobre la relación entre los eventos externos (lo que el paciente dice, o hace) y los estados mentales internos que pueden inferirse de ellos, así como sobre sus propios estados internos. En la relación con el paciente, el terapeuta debe tratar de mantenerse en el "aquí y ahora» de la relación terapéutica, favoreciendo que el paciente explore e identifique las emociones asociadas.

b) Tender puentes entre los vacíos: existe un "vacío» entre la experiencia afectiva primaria y su representación en los pacientes con TLP, que da lugar a su característica impulsividad. Se trata de favorecer los procesos reflexivos, ayudando al paciente a que integre las experiencias y les de un significado, para que pueda acomodarlas a su narrativa. No se trata de darle interpretaciones complejas, sino de revelarle cómo el terapeuta cree que el paciente está viviendo la relación terapéutica. El objetivo es que el paciente sienta que su mente está siendo explorada por otra mente, que sienta que el terapeuta tiene «su mente en mente». La relación será vista como un «lugar» en el que se puede «jugar con las ideas»

c) Trabajar con estados mentales actuales: No centrarse en el pasado continuamente, sino tratar de dilucidar cómo los estados mentales actuales pueden estar siendo influidos por los eventos pretéritos. Hay que reorientar todas las emociones fuertes relacionadas con el pasado hacia el presente, para aprender a manejarlas en el aquí y el ahora. d) Tener en cuenta los déficit del paciente: en ocasiones, los pacientes parecen funcionar adecuada y sofisticadamente. Sin dejar de fijarnos en sus potencialidades, es importante tener en cuenta sus dificultades para evitar, por ejemplo, violaciones de los límites o interpretaciones inadecuadas por parte del paciente.

Remitimos al lector interesado en profundizar en aplicación de las técnicas específicas para favorecer la mentalización a la guía de tratamiento de Bateman y Fonagy (2006). Como hemos dicho anteriormente, éstas se organizan en función de su profundidad y deben aplicarse en diferentes momentos de la terapia. En líneas generales, el tipo de intervención está inversamente relacionada con intensidad emocional que presente el paciente en ese momento. Es decir, si el paciente se ve desbordado por la emoción, es mejor quedarse en el nivel de «apoyo»; si vemos que es capaz de contener los afectos, podemos ir profundizando para avanzar en la mentalización. El «espectro» de intervenciones con el que contamos (de menor a mayor profundidad) es el siguiente:

- Reaseguración, apoyo y empatía.

- Clarificación, desafío y elaboración de los afectos.

- Mentalización básica: dirigidas a reinstaurar la mentalización cuando ésta se ha perdido. Los autores organizan estas técnicas en dos grupos: "párate, escucha, mira» («stop, listen, look») y «párate, rebobina, explora» («stop, rewind, explore»). La primera consiste en mantener la sesión "en suspenso» mientras se investiga lo que está sucediendo en ese momento. La segunda trata de analizar hacia atrás, una vez se ponen de manifiesto conductas/verbalizaciones que apuntan a un fallo en la mentalización, toda la secuencia de hechos que nos han llevado hasta allí, para reflexionar conjuntamente sobre lo que ha pasado.

- Mentalización interpretativa: un paso más allá, que debe hacerse con cautela. El terapeuta ofrece una perspectiva al- 
ternativa sobre lo que el paciente dice, relacionando la reacción del paciente a un estado mental, en una secuencia causal.

- Mentalización de la transferencia: consiste en animar al paciente a pensar sobre la relación con el terapeuta en el momento actual, para que centre su atención en la mente del otro, y para ayudarle a contrastar su propia percepción sobre cómo es visto por el otro.

En todo momento, el terapeuta procura mantener el foco en la mentalización, explicando al paciente los estados mentales que infiere de él a partir de sus conductas, aceptando que la mente del paciente no es $100 \%$ accesible para él y que puede errar en sus interpretaciones. No se trata de dar al paciente interpretaciones hechas, sino de estimular el análisis conjunto de las situaciones para llegar a conclusiones lo más consensuadas posible. En definitiva, se trata de trabajar «codo con codo» con el paciente para lograr el fin último, es decir, percibirse como un sujeto «agente» detrás de cuyas conductas existen una serie de deseos, creencias, pensamientos $\mathrm{y}$ afectos que le son propios, que surgen de su propia mente. Sólo así podrá sentirse dueño de sí.

\section{Conclusiones}

Hemos intentado presentar, a modo introductorio, un modelo de psicoterapia psicodinámica desarrollado específicamente para el TLP que se ha demostrado efectivo en varios ensayos controlados aleatorizados, tanto en su modalidad de hospitalización parcial, como en el tratamiento intensivo ambulatorio (Bateman y Fonagy, 1999, 2001, 2008, 2009, 2010), en la reducción de conductas suicidas y autolesivas, así como en la mejoría del funcionamiento social e interpersonal, por encima de otros tratamientos convencionales. Además, muchos de dichos logros pueden llegar a mantenerse en los seguimientos a 18 meses (Bateman y Fonagy, 2001) y a los ocho años (Bateman y Fonagy, 2008). Es difícil resumir en unas pocas páginas el espíritu que subyace a este modelo, tanto en su manera de entender el desarrollo del sentido del self, como en su comprensión sobre lo que «funciona mal» en el TLP y en cómo abordarlo. Para Bateman y Fonagy (2004) es fundamental la creación de un contexto de apego seguro en el que se puede explorar libremente la mente del otro para encontrar la mente propia representada en ella. Y esto es una máxima que podemos (y en nuestra opinión, debemos) aplicar los profesionales en el tratamiento con estos pacientes, independientemente de nuestro enfoque terapéutico. De ahí la grandeza del trabajo de Anthony Bateman y Peter Fonagy.

\section{Referencias}

Allen, J. G y Fonagy, P. (2006). Handbook of Mentalization-Based Treatment. New York: Wiley.

Bateman, A. y Fonagy, P. (1999). Effectiveness of partial hospitalization in the treatment of borderline personality disorder: a randomized controlled trial. American Journal of Psychiatry, 156, 1562-1569.

Bateman, A. y Fonagy, P. (2001). Treatment of borderline personality disorder with psychoanalytically oriented partial hospitalization: an 18-month follow-up. American Journal of Psychiatry, 158, 36-42.

Bateman, A. y Fonagy, P. (2004). Psychotherapy for Borderline Personality Disorder: mentalizationbased treatment. Nueva York: Oxford University Press.

Bateman, A. y Fonagy, P. (2006). Mentalizationbased treatment for Borderline Personality Disorder: a practical guide. Nueva York: Oxford University Press.

Bateman, A. y Fonagy, P (2008). 8-year follow-up of patients treated for Borderline Personality Disorder: mentalization-based treatment versus treatment as usual. American Journal of Psychatry, 165, 631-638.

Bateman, A. y Fonagy, P. (2009). Randomized controlled trial of outpatient mentalization-based treatment versus structure clinical management for borderline personality disorder. American Journal of Psychiatry, 166, 1355-1364. 
Bateman, A. y Fonagy, P. (2010). Mentalization based treatment for borderline personality disorder. World Psychiatry, 9, 11-15.

Bouchard, M. A., Lecours, S., Tremblay L. M., Target, M., Fonagy, P. y Schachter, A. (2008). Mentalization in adult attachment narratives: reflective functioning, mental states, and affect elaboration compared. Psychoanalitic Psychology 25(1), 47-66.

Bowlby, J. (1998). El apego [Attachment]. Madrid, España: Paidós.

Clarkin, J. F., Yeomans, F. E. y Kernberg, O.F. (2006). Psychotherapy for Borderline Personality. Focusing on Object Relations. Arlington, VA: American Psychiatric Publishing.

Cuevas, C. y López, A. G. (2012). Intervenciones psicológicas eficaces para el tratamiento del trastorno límite de la personalidad [Effective psychological interventions for the treatment of borderline personality disorder]. International Journal of Psychology and Psychological Therapy, 12(1), 97-114.

Fonagy, P. (1991). Thinking about thinking: some clinical a theoretical considerations in the treatment of a borderline patient. International Journal of Psychoanalysis, 72, 1-18.

Fonagy, P., Leigh, T., Steele, M., Steel, H., Kennedy, R., Mattoon G.,... y Gerber, A. (1996). The relationship of attachment status, psychiatric classification, and response to psychotherapy. Journal of Consulting and Clinical Psychology, 64, 2-31.

Fonagy, P., Target, M. y Gegerly, G. (2000). Attachment and Borderline Personality Disorder: a theory and some evidence. Psychiatric Clinics of North America, 23(1), 103-122.

Fonagy, P., Target, M.,, Steele, H. y Steele, M. (1997). Reflective functioning manual for application to Adult Attachment Interviews. Londres, UK: University College London.

Fonagy, P. y Target, M. (1996). Playing with reality: I. Theory of mind and the normal development of psychic reality. International Journal of Psychoanalysis, 77, 217-233.
Gegerly, G. y Watson, J. (1996), The social biofeedback model of parental affect mirroring. International Journal of Psychoanalysis. 77, 1181-1212.

George, C., Kaplan, N. y Main, M. (1985). The Adult Attachment Interview. Unpublished manuscript. University of California, Berkeley.

Gunderson, J. G. y Lyons-Ruth, K. (2008). Borderline Personality Disorder's interpersonal hypersensitivity phenotype: A gene-environmentdevelopmental model. Journal of Personality Disorders, 22(1), 22-41.

Keinänen, M. T., Johnson, J. G., Richards, E. S. y Courtney, E. S. (2012). A systematic review of the evidence-based psychosocial risk factors for understanding of borderline personality disorder. Psychoanalytic Psychotherapy, 26(1), 65-91.

Kernberg, O. F. (1999). Trastornos Graves de la Personalidad [Severe Personality Disorders]. México, D.F.: Manual Moderno.

Lanza Castelli, G. (2011, febrero). Mentalización: aspectos teóricos y clínicos [Mentalization: theoretical and clinical aspects]. Trabajo presentado en el XII Congreso Virtual de Psiquiatría Interpsiquis, España.

Lyons-Ruth, K. (1996). Attachment relationships among children with aggresive behavior problems: the role of disorganized early attachment patterns. Journal of Consultant and Clinical Psychology, 64, 64-73.

Mas, J. (2012, abril). La Terapia Basada en la Mentalización para el TLP. [The Mentalizationbased treatment for BPD] Comunicación presentada en el curso Aula Complutense de la Personalidad: talleres terapéuticos. Madrid, España.

Nickell, A. D., Waudby, C. J. y Trull, T. J. (2002). Attachment, Parental Bonding and Borderline Personality Disorder Features in Young Adults. Journal of Personality Disorders, 16(2), 148-159.

Winnicott, D. W. (1982). Realidad y juego [Playing and reality]. Barcelona, España: Gedisa. 
